\title{
Robot-Assisted Reconstruction in Head and Neck Surgical Oncology: The Evolving Role of the Reconstructive Microsurgeon
}

\author{
Richard Chalmers $^{\mathrm{a}} \quad$ Juergen Schlabe ${ }^{\mathrm{a}} \quad$ Elizabeth Yeung $^{\mathrm{a}} \quad$ Cyrus Kerawala $^{\mathrm{b}}$ \\ Luke Cascarini $^{a}$ Vinidh Paleri ${ }^{b}$ \\ a'Guy's and St Thomas' Hospital NHS Foundation Trust, London, UK; \\ bThe Royal Marsden NHS Foundation Trust, London, UK
}

\section{Keywords}

Robotics · Head and neck surgery · Reconstructive microsurgery

\begin{abstract}
Transoral robotic surgery (TORS) is gaining more widespread use among head and neck surgical procedures. As experience grows with this technique, so do the indications of when and in which patients it can be used. Already established in the treatment of small oral cavity tumours, it is expanding into larger multi-site resections and resections, such as through-andthrough-into-the-neck defects, that will require reconstruction. With robot-assisted surgery advancing, so robot-assisted reconstruction (RAR) is evolving. In this paper, we discuss the evolving role of reconstruction in post-TORS defects as well as the role of RAR in today's practice.

(c) 2018 S. Karger AG, Basel
\end{abstract}

\section{Introduction}

As technology advances, so do the opportunities that present themselves to clinicians to improve the delivery of care to patients. Robot-assisted surgery (RAS) has been in use for over 30 years. The first RAS documented was performed in 1985 using the Puma 560 robotic arm for a neurosurgical biopsy. The same robotic system went on to perform the first laparoscopic robot-assisted cholecystectomy in 1987 [1]. By 2000, the most common robotic system in use, the da Vinci Surgical System (Intuitive Surgical, Sunnyvale, CA, USA), had gained Food and Drug Administration (FDA) approval for general laparoscopic surgery [2]. In the fields of urology, gynaecology and general surgery, RAS is becoming more commonplace, with up to $80 \%$ of all radical prostatectomies in the USA being performed robotically [3]. 
Table 1. Benefits of RAR or TORRS

Improved visualisation especially in a confined working space

High-resolution and high-magnification images

Instrumentation accuracy and reduction of tremor

Improved optics allowing for microsurgical anastomosis

Improved access to oropharyngeal tumours involving the tonsils, base of tongue and soft palate

Preserves the concept of minimising scars or morbidity from mandibular lip split procedures

Increasing indication in salvage or recurrent cases as experience grows

As more research into the benefits of TORS emerges, so researchers are also gaining experience in

providing minimal-access reconstructive options such as TORRS

RAR, robot-assisted reconstruction; TORRS, transoral robotic reconstructive surgery; TORS, transoral robotic surgery.

Application of RAS in head and neck surgery is also gaining momentum. With the drive to reduce morbidity from larger resections requiring mandibular split procedures for access and to reduce the complications from chemoradiotherapy (CRT) [4, 5], transoral robotic surgery (TORS) for the surgical management of oropharyngeal tumours was developed.

Transoral robotic oropharyngeal resections have traditionally been left to heal by secondary intention. As more experience is gained in the use of robotic systems in head and neck surgery, surgeons are assessing the application of TORS in larger, more complex resections. These defects often cross various anatomical subsites and as a result require reconstruction. Goals for reconstruction include coverage of vital vascular structures, minimising velopharyngeal insufficiency, maintaining a water-tight seal between the oropharynx and the neck for through-and-through resections, restoring the tongue volume and minimising longterm functional deficits. This is achieved by the transfer of vascularised tissue either locally or more often as a microvascular free flap [6-8].

Reconstructive algorithms have been developed to aid planning of post-TORS resection defects. A system suggested by de Almeida et al. [7] has four classes of defect: class I involves one subsite (tonsil, tongue base, pharynx or soft palate) and no adverse features (internal carotid artery exposure, neck communication or $>50 \%$ of soft palate resection); class II is similar but involves more than one subsite of the oropharynx; class III involves one subsite but has one or more adverse features; and class IV involves multiple subsites and one or more adverse features. The authors recommend that class I and II defects should be either left to heal by secondary intention or local flaps, whilst class III or IV defects require regional or free flap reconstruction. In terms of a reconstructive strategy following TORS resections, reconstruction should be planned if coverage of major vessels is needed or if a substantial throughand-through defect into the neck occurs [9]. Numerous local flaps have been described for post-TORS defects depending upon the site of resection. Local flaps include the facial artery musculomucosal flap, the infrahyoid myocutaneous flap, the nasoseptal flap after tonsillectomy and distant flaps ranging from temporalis transfers to more traditional pectoralis major flaps [10].

As experience improves, so larger and more complex resections, including salvage surgery, are becoming possible with TORS $[9,11,12]$. The natural evolution of this process has meant that reconstructive surgeons are beginning to investigate the role of robot-assisted reconstruction (RAR) and transoral robotic reconstructive surgery (TORRS) [13]. With the use of TORS growing steadily, the application of RAR becomes more appealing. Aside from being a more cost-effective and efficient use of the robotic system, if both resection and reconstruction can be performed during the same procedure, authors have cited other benefits 
(Table 1). One of the major benefits of TORS and TORRS is the avoidance of mandibulotomy for access. In the short term, a reduced operation time and length of hospital stay as well as a quicker return of swallow have been reported [14]. In the long term, a reduction in primary CRT and de-escalation of postoperative CRT through TORS pathways may reduce the incidence of osteoradionecrosis, which carries a large economic burden and has a significant impact on quality of life [15-17]. It should be pointed out that there is a steep learning curve for the surgeon performing TORS, and managing complex and salvage cases should not be undertaken early on in this process [9].

RAR has been described for use in three areas in head and neck surgery: flap inset, flap harvest and vascular anastomosis.

\section{Robot-Assisted Flap Inset}

In the first RAR series of oropharyngeal defects, Selber [18] describes 5 cases undergoing oropharyngeal resections with an intact mandible. This series demonstrates well a number of applications for RAR in the clinical setting. Two cases underwent TORS resection and reconstruction with a free radial forearm flap in one case, and a facial artery musculomucosal (FAMM) flap in the other. The radial forearm reconstruction was completely inset, with the robot reconstructing a defect involving the floor of mouth, base of tongue and tonsillar fossa. The FAMM flap reconstruction was raised in the standard way and inset with the robot. It reconstructed a small defect involving the posterior tonsillar pillar, soft palate and posterior pharynx with exposure of the carotid artery. In a unique application of RAS, the patient unfortunately bit through the pedicle at day 2 and underwent debridement of the flap robotically.

Selber [18] goes on to describe another salvage case whereby part of the flap reconstruction was inset using the robot. A double-paddled anterolateral thigh flap (ALT) was used to resurface the neck and reconstruct the tongue, floor of mouth and pharyngeal defects. The anterior inset was performed by hand through the mouth, whilst the distal portion of the pharyngeal inset was performed by hand through the pharyngotomy. The inset in-between which could not be performed by hand was completed robotically. In another case a pullthrough resection from the base of tongue and a 2-cm-wide mucosal defect from the floor of mouth to vallecula was closed primarily. In the final case a tongue and floor of mouth defect was reconstructed with a free ALT flap. Neck access was adequate enough for the inset to be performed by hand. In the neck, whilst the venous anastomosis was performed with loupes and a coupler, the artery (superior thyroid artery to descending branch of the lateral circumflex femoral artery) was sutured using the robot. In this case three robotic arms and two Black Diamond robotic microneedle drivers were used (Fig. 1). The 0 and $180^{\circ}$ were performed first, followed by the back wall and then the front wall. No complications were reported. They describe, however, that even in some restricted positions, the suture was placed robotically but needed to be hand tied.

A clear indication for the use of RAR is in insetting the flap in anatomical areas that are often difficult to access (Fig. 2). Chan et al. [19] describe a total pharyngolaryngectomy with a distal open approach combined with a TORS approach for the superior resection. The tumour was delivered transorally. The reconstruction using a free jejunal flap was delivered transorally to the neck, and the proximal anastomosis between the jejunum and oropharynx was performed robotically. The authors identify that the jejuno-oropharyngeal anastomosis would not have been possible without the da Vinci robotic system due to limited surgical access and that the robotic system allowed the accurate placement of sutures due to the superior optics at the lateral pharyngeal wall and tongue base. 


\section{\begin{tabular}{l|l}
\hline DOI: $10.1159 / 000492787$ & (c) 2018 S. Karger AG, Basel
\end{tabular} www.karger.com/orl}

Chalmers et al.: Robot-Assisted Reconstruction in Head and Neck Surgical Oncology

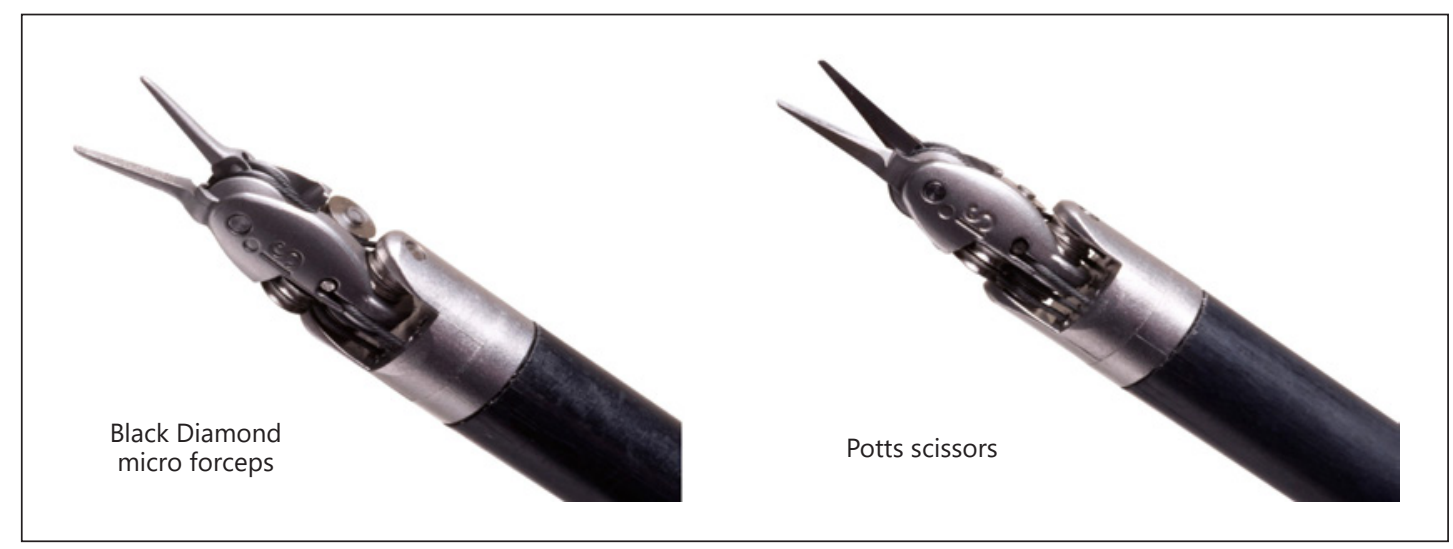

Fig. 1. Black Diamond micro forceps and Potts scissors (from da Vinci Xi Instrument \& Accessory catalogue 2015).
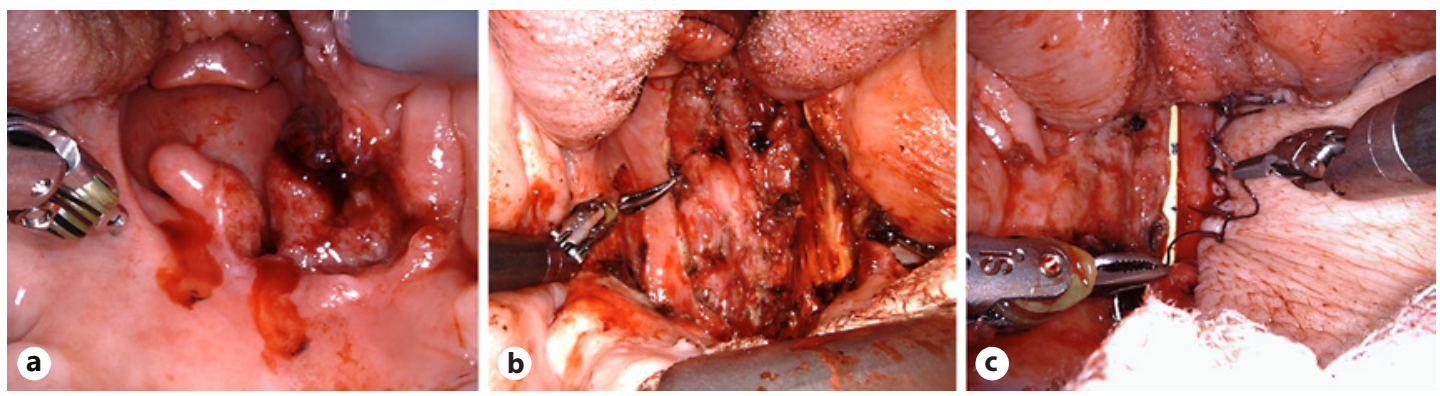

Fig. 2. Example of robot-assisted inset. Patient with T3N1 squamous cell carcinoma after chemoradiotherapy. a Tumour involving the right tonsillar fossa, extending medially into the soft palate up to the midline and inferiorly into the tongue base and the lateral pharyngeal wall. b Post-transoral robotic surgery resection defect, where the resection involved the soft palate and the full thickness of the constrictor muscles. Note that the parapharyngeal space fat and prevertebral fascia are exposed, with the lower extent of the defect at the level of the hyoid bone. c Robotic inset of a free radial forearm flap. Pictures courtesy of V.P.

Gorphe et al. [20] describe their technique for salvage oropharyngectomies using both a cervical approach and a TORS approach. They do not report on a series of patients but highlight that the superior flap inset is easily accessed via the oral route, whilst the inferior inset is performed robotically. Of note they use a very thin suprafascial free ALT for reconstruction in these cases. This is an important feature of TORRS reconstruction, in that in a limited space, the thinner the flap, the better. In addition, they identify that the inset is performed by hand initially, then the robotic system is reinstalled to complete the inset. The timing of the instalment and removal of the robot was not commented upon.

Mukhija et al. [21] describe 2 cases that underwent TORS excision of a soft palate and tonsillar lesion and a salvage case involving the retromolar space, palate and oropharynx. Inset of the radial forearm flaps was performed robotically. Both cases took approximately $4 \mathrm{~h}$, and the authors report that the room setup time was $10 \mathrm{~min}$. Positive comments from the authors include that the TORS approach avoids a mandibular split, reducing morbidity and reducing the operative time by $2-3 \mathrm{~h}$. As a result the hospital stay was shortened by approximately 3 days. The authors report successful oncological resections and excellent recovery of speech and swallow. 
Paleri et al. [9] describe their approach to RAR in a group of 26 patients undergoing TORS for residual and recurrent oropharyngeal cancers. The average times to de-cannulation and re-instatement of oral diet were 5.8 and 13 days, respectively. The average inpatient stay was 13.4 days (unpubl. data; provided by V.P.). Three patients had defects reconstructed with free flaps that were inset using robotic assistance. Thin fasciocutaneous flaps were used - two radial forearm free flaps and one medial sural artery perforator flap. The defect was mapped out and contoured and the vascular pedicle was passed into the neck within the lumen of a Penrose drain. Anastomosis was performed to the superior thyroid artery and the internal jugular vein. It was noted that the added time to the procedure was mainly due to lack of availability of 5-mm needle drivers. The 8-mm drivers take up limited space and reduce manoeuvrability. The development of reconstructive instrumentation in the future will help in this regard.

\section{Robot-Assisted Flap Raising}

Aside from flap insets, other areas for the use of robotic systems in reconstruction have been explored. In the plastic surgery literature, flaps for breast reconstruction, such as the latissimus dorsi and the rectus abdominis flap for lower limb and perineal defects, have been described [22-25]. The main benefit identified is in minimising the extent of the scar during flap harvest. A limitation in translating this to head and neck cases is that simultaneous resection and flap raising by two teams is not possible without two teams and two robotic systems. This would add further time and inefficiency to the procedure. Local intraoral flaps, such as the FAMM flap already described, allow resection and reconstruction to be performed in sequence, which is a practical use of the technology.

In a series of FAMM flaps for small soft palate TORS resections, Bonawitz and Duvvuri [26] demonstrate that the raising and inset of this flap is possible. They do present their complications, which includes flap dehiscence in 2 out of 5 cases, requiring further treatment. Whether this is a patient-related, flap-related factor or whether robotic inset is indeed prone to dehiscence is something to bear in mind when considering using this technique. The average time for the procedures was 6-10 h. The authors identify that the delays were in setting up for the robotic procedure and docking, and not in the raising and insetting of the reconstruction. As this setup becomes more efficient, operative times for these local flap reconstructions should improve.

\section{Robot-Assisted Anastomosis}

An interesting area developing is the role of robotics in microsurgery. With reduction of tremor, the development of finer microsurgical robotic instrumentation and the promise of improved optics, robotic microvascular anastomosis may become more commonplace in the years to come.

Selber [18] presented in his case series 1 case where the arterial anastomosis was performed robotically. Setup and the venous anastomosis were performed by hand through a wide traditional neck incision. Song et al. [6] presented a series of 5 patients that underwent a combination of RAS. Three cases underwent TORS resection, 3 cases underwent robotassisted flap inset and 4 underwent robot-assisted neck dissection via a limited retroauricular incision. Here, the use of the robotic system was put to good use to limit the scar to the postauricular region, avoiding the traditional scar on the neck towards the midline. To minimise the need to extend the short postauricular incision, 1 patient underwent robotic 
Table 2. Potential limitations of TORRS

Cost

Time and efficiency

No haptic feedback currently, which is essential for microsurgery

Safety evidence lacking

Not amenable to two-team operating in a TORS setting when both teams need the robot at the same time

Steep learning curve required

No evidence that it improves outcomes

Unknown number of cases to warrant investment in a TORRS reconstructive programme

TORRS, transoral robotic reconstructive surgery; TORS, transoral robotic surgery.

microvascular anastomosis to the facial artery. It is not clear whether the venous system was also anastomosed with the robot. The authors report the anastomosis took $150 \mathrm{~min}$ to complete.

Lai et al. [27] report a series of 5 patients undergoing oropharyngeal reconstruction following resection. In all cases the reconstruction was with a radial forearm free flap. The artery and one vena comitans were anastomosed by hand. The second vena comitans was anastomosed using the robot. The time taken for the robotic anastomosis was $40 \mathrm{~min}$, whilst the inset time on average was $31 \mathrm{~min}$. The authors state the importance of specific microsurgical training in robotic reconstruction. Visual cues when tying knots, alongside an understanding of vessel manipulation and cutting of suture knots, are different to conventional microsurgery techniques. Numerous authors have identified the feasibility and benefit of robotic microsurgical training for both the robotically trained surgeon and the trained microsurgeon [28-30]. As the indications for robot-assisted microsurgery increase and instrumentation and technology move forward, specific training schemes to remain up to date will become more necessary.

\section{Limitations of RAR}

Numerous limitations have been mentioned in the literature (Table 2). One of the main limitations commonly discussed is the cost related to each procedure, be that the up-front cost and setup or the cost per case [3]. However, the cost savings over potential long-term morbidity associated with osteoradionecrosis can be significant. Additionally, only one EndoWrist ${ }^{\mathrm{TM}}$ instrument needs to be added to the expense sheet, which in most instances will amount to approximately GBP 500. As RAR is in its infancy, studies looking at outcomes and timings for reconstructive options are not available yet. Added costs of staff and specialist equipment when performing RAR have also not been calculated. Most importantly, there are no studies that show improved outcomes and safety when performing RAR over conventional methods. However, the efficient use of the robot in performing more steps in complex head and neck procedures may improve the cost-effectiveness of the robotic system and promote its use further.

Access and use of the robot to inset a flap in difficult anatomical sites appears to be the primary benefit cited in the literature. In a series of 42 consecutive TORS patients that underwent free flap reconstruction, Hatten et al. [31] state that robotic instrumentation was not required to aid flap inset for any of their cases. It is clear that robot-assisted inset is not indicated in all cases, and that in the majority of standard TORS cases, robotic assistance is 
not needed. The role for RAR may not be with the current "typical" TORS cases but with the larger, more challenging salvage cases as experience with TORS techniques growths and larger resections are undertaken using the robot.

Aside from flap inset, assisted microvascular anastomosis shows promise. With improved optics and magnification there may come a time that a robotic system replaces the operating microscope and all microsurgery can be performed robotically. Before that time, considerations concerning the safety of such systems, including techniques to manage intraoperative complications such as a vessel tear, anastomotic leak or haemorrhage, must be taken into account. Further rigorous laboratory and clinical studies are needed.

RAS is becoming a valuable tool in a number of specialties. Its role in head and neck surgery, and especially in reconstructive surgery, is still being defined. Flap inset is the most obvious role for the robotic system in reconstructive surgery at present. As technology advances, so do the opportunities it offers to use improved techniques and precision in the operating room. Within the framework of well-designed trials alongside TORS, it may also be shown to improve overall outcomes for our patients.

\section{Disclosure Statement}

The authors have no conflicts of interest to disclose.

\section{References}

1 Ballantyne GH: Robotic surgery, telerobotic surgery, telepresence, and telementoring. Review of early clinical results. Surg Endosc 2002;16:1389-1402.

2 Shah J, Vyas A, Vyas D: The history of robotics in surgical specialties. Am J Robot Surg 2014;1:12-20.

3 Barbash GI, Glied SA: New technology and health care costs - the case of robot-assisted surgery. N Engl J Med 2010;363:701-704.

4 Wolf GT, Fisher SG, Hong WK, Hillman R, Spaulding M, Laramore GE, et al: Induction chemotherapy plus radiation compared with surgery plus radiation in patients with advanced laryngeal cancer. N Engl J Med 1991; 324:1685-1690.

5 Campbell BH, Spinelli K, Marbella AM, Myers KB, Kuhn JC, Layde PM: Aspiration, weight loss, and quality of life in head and neck cancer survivors. Arch Otolaryngol Head Neck Surg 2004;130:1100-1103.

6 Song HG, Yun IS, Lee WJ, Lew DH, Rah DK: Robot-assisted free flap in head and neck reconstruction. Arch Plast Surg 2013;40:353-358.

7 de Almeida JR, Park RC, Villanueva NL, Miles BA, Teng MS, Genden EM: Reconstructive algorithm and classification system for transoral oropharyngeal defects. Head Neck 2014;36:934-941.

8 de Almeida JR, Park RC, Genden EM: Reconstruction of transoral robotic surgery defects: principles and techniques. J Reconstr Microsurg 2012;28:465-472.

9 Paleri V, Fox H, Coward S, Ragbir M, McQueen A, Ahmed O, et al: Transoral robotic surgery for residual and recurrent oropharyngeal cancers: exploratory study of surgical innovation using the IDEAL framework for early-phase surgical studies. Head Neck 2018;40:512-525.

10 Meccariello G, Montevecchi F, Sgarzani R, De Vito A, D’Agostino G, Gobbi R, et al: The reconstructive options for oropharyngeal defects in the transoral robotic surgery framework. Oral Oncol 2017;66:108-111.

11 White H, Ford S, Bush B, Holsinger FC, Moore E, Ghanem T, et al: Salvage surgery for recurrent cancers of the oropharynx: comparing TORS with standard open surgical approaches. JAMA Otolaryngol Head Neck Surg 2013;139:773-778.

12 Dabas S, Dewan A, Ranjan R, Dewan AK, Shukla H, Sinha R: Salvage transoral robotic surgery for recurrent or residual head and neck squamous cell carcinoma: a single institution experience. Asian Pac J Cancer Prev 2015;16:7627-7632.

13 Selber JC: Can I make robotic surgery make sense in my practice? Plast Reconstr Surg 2017;139:781e-792e.

14 Lee SY, Park YM, Byeon HK, Choi EC, Kim SH: Comparison of oncologic and functional outcomes after transoral robotic lateral oropharyngectomy versus conventional surgery for T1 to T3 tonsillar cancer. Head Neck 2014; 36:1138-1145.

15 Pang L, Jeannon JP, Simo R: Minimizing complications in salvage head and neck oncological surgery following radiotherapy and chemo-radiotherapy. Curr Opin Otolaryngol Head Neck Surg 2011;19:125-131. 
16 Patel V, Ormondroyd L, Lyons A, McGurk M: The financial burden for the surgical management of osteoradionecrosis. Br Dent J 2017;222:177-180.

17 Park L, Lilic N, Addison B, Patel R: Cost analysis of osteoradionecrosis. J Laryngol Otol 2017;131:303-308.

18 Selber JC: Transoral robotic reconstruction of oropharyngeal defects: a case series. Plast Reconstr Surg 2010; 126:1978-1987.

19 Chan JYW, Chan RCL, Chow VLY, Tsang RKY, Wong STS, Wei WI: Transoral robotic total laryngopharyngectomy and free jejunal flap reconstruction for hypopharyngeal cancer. Oral Oncol 2017;72:194-196.

20 Gorphe P, Temam S, Kolb F, Qassemyar Q: Cervical-transoral robotic oropharyngectomy and thin anterolateral thigh free flap. Eur Ann Otorhinolaryngol Head Neck Dis 2018;135:71-74.

21 Mukhija VK, Sung CK, Desai SC, Wanna G, Genden EM: Transoral robotic assisted free flap reconstruction. Otolaryngol Head Neck Surg 2009;140:124-125.

22 Chung JH, You HJ, Kim HS, Lee BI, Park SH, Yoon ES: A novel technique for robot assisted latissimus dorsi flap harvest. J Plast Reconstr Aesthet Surg 2015;68:966-972.

23 Clemens MW, Kronowitz S, Selber JC: Robotic-assisted latissimus dorsi harvest in delayed-immediate breast reconstruction. Semin Plast Surg 2014;28:20-25.

24 Pedersen J, Song DH, Selber JC: Robotic, intraperitoneal harvest of the rectus abdominis muscle. Plast Reconstr Surg 2014;134:1057-1063.

25 Selber JC, Baumann DP, Holsinger FC: Robotic latissimus dorsi muscle harvest: a case series. Plast Reconstr Surg 2012;129:1305-1312.

26 Bonawitz SC, Duvvuri U: Robotic-assisted FAMM flap for soft palate reconstruction. Laryngoscope 2013;123: 870-874.

27 Lai CS, Chen IC, Liu SA, Lu CT, Yen JH, Song DY: Robot-assisted free flap reconstruction of oropharyngeal cancer - a preliminary report. Ann Plast Surg 2015;74(suppl 2):S105-S108.

28 Clarke NS, Price J, Boyd T, Salizzoni S, Zehr KJ, Nieponice A, et al: Robotic-assisted microvascular surgery: skill acquisition in a rat model. J Robot Surg 2018;12:331-336.

29 Selber JC, Alrasheed T: Robotic microsurgical training and evaluation. Semin Plast Surg 2014;28:5-10.

30 Liverneaux PA, Hendriks S, Selber JC, Parekattil SJ: Robotically assisted microsurgery: development of basic skills course. Arch Plast Surg 2013;40:320-326.

31 Hatten KM, Brody RM, Weinstein GS, Newman JG, Bur AM, Chalian AA, et al: Defining the role of free flaps for transoral robotic surgery. Ann Plast Surg 2018;80:45-49. 